\title{
A computer aided tolerancing tool I: Tolerance specification
}

\author{
O.W. Salomons * , H.J. Jonge Poerink, F.J. Haalboom, F. van Slooten, \\ F.J.A.M. van Houten, H.J.J. Kals
}

University of Twente, Department of Mechanical Engineering, Laboratory of Production and Design Engineering, P.O. Box 217, 7500 AE Enschede, The Netherlands

Received 16 August 1995; revised 29 February 1996; accepted 4 July 1996

\begin{abstract}
In current tolerancing practice, designers have to manually specify tolerances: either on a drawing or in a CAD system. Different designers will possibly arrive at different tolerance specifications for the same nominal geometry. The paper demonstrates that this situation can be avoided in the case of functional tolerancing with a focus on the geometry relevant for functioning. Under this restriction, the specification of too tight or too many tolerances can also be avoided. The paper describes a tool for functional tolerance specification which supports the user in automatically proposing geometric tolerance types where the user only has to give in the tolerance values. Apart from this semi-automatic tolerance type specification, manual specification is still possible.
\end{abstract}

Keywords: Tolerance specification; Tolerance representation; TTRS

\section{Introduction}

This paper is the first in a series of two on a computer aided tolerancing tool as part of a larger academic prototype focusing on re-design support in general called FROOM. This paper elaborates on computer support of the specification of tolerances whereas the second paper focuses on tolerance analysis. Tolerance specification is introduced in Section 1.1. The FROOM system is introduced in Section 1.2. Section 1.3 provides an overview of the remainder of the paper.

\subsection{Tolerance specification}

Tolerance specification is the activity of specifying tolerances; defining the tolerance types and related tolerance values (geometric tolerances are assumed). Tolerance specification is preferably carried out in conformance with the tolerancing standards (e.g. ISO 1101 [1], ANSI Y14.5M [2]). However, the standards do not give a method of how tolerances should be specified. Therefore, research into the specification of tolerances seems to be required, especially because only relatively few research in this field has been performed so far.

\footnotetext{
${ }^{*}$ Corresponding author. Email: o.w.salomons@wb.utwente.nl
} 


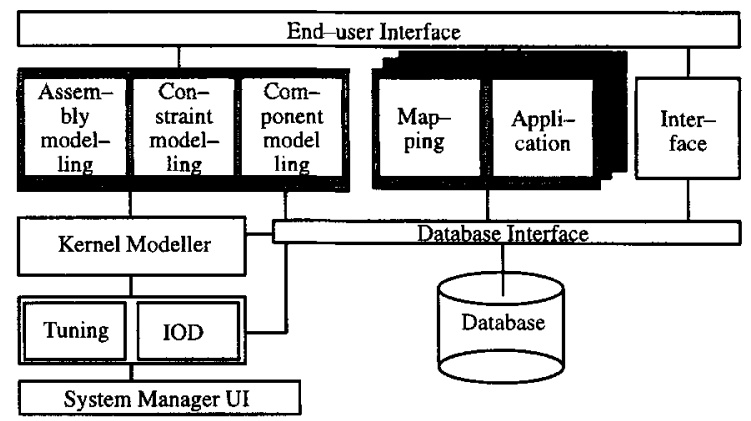

Fig. 1. System architecture of FROOM (IOD = interactive object definition).

In current tolerancing practice, designers have to manually specify both tolerance types and values either on a drawing or in a CAD system. The tolerance specification in this case depends largely on the designer's judgement and experience regarding subjects like functioning, materials and manufacturing processes. Therefore, different designers will possibly arrive at different tolerance specifications for the same nominal geometry.

\subsection{Froom}

FROOM is a prototype of a re-design support system, currently under development. FROOM stands for Features and Relations used in Object Oriented Modeling. FROOM incorporates tolerancing functionality aiming to support (re-)design and to integrate with CAPP. FROOM is a feature based system, allowing the modeling of both components and assemblies. FROOM employs conceptual graphs for knowledge representation [3]. Fig. 1 shows FROOM's system architecture.

Two different kinds of users are distinguished: end-users and system manager users. For these two different user groups, separate user interfaces are available. End-users are the designers or draftsmen who design and detail assemblies, components, etc.; they perform the actual design tasks. In the modeling module of FROOM, components, assemblies and constraints can be modeled by the end-user. The modeling module has access to the kernel modeler, which offers the basic geometry processing functionality. In FROOM, the commercially available ACIS ${ }^{\mathrm{TM}}$ kernel modeler is used for this [4]. The tolerancing tool is part of the (constraint) modeling module of FROOM. The application module includes the possible mappings to applications and the applications themselves. For more details on FROOM, see [3,5,6]. System manager users customize the system to a certain application domain, company and user (group). They define the features to be used, the catalogues from which selections can be made, etc. For the tolerancing tool end-users are of main interest.

\subsection{Overview of the paper}

An overview is provided of previous work in tolerance specification and representation in Section 2 . In Section 3 the overall design of the tolerance specification functionality in FROOM is presented. The TTRS based tolerance representation that is employed is addressed in Section 4. Semi-automatic tolerance specification is briefly elaborated in Section 5 while manual tolerance specification is addressed in Section 6. Finally, conclusions and recommendations are provided in Section 7.

\section{Previous work}

Tolerance representation is important together with tolerance specification as the way tolerances are represented often influences the way in which they can be specified and vice versa. An adequate tolerance 
representation enables computerization of applications following tolerance specification such as tolerance analysis and tolerance synthesis. A subdivision is therefore made into tolerance representation (Section 2.1), and tolerance specification (Section 2.2).

\subsection{Previous work in tolerance representation}

A lot of (possibly theoretically and mathematically correct) approaches for the computer representation of tolerances as proposed in literature do not sufficiently comply with the international standards. For human designers, these approaches will not be sufficient to replace the tolerancing standards as they are not expressive enough; their "syntax and scmantics" arc too limited. Morcover, it will be hard to introducc a completely new and different tolerancing standard on a world wide basis even if it has sufficient expressiveness to humans as well as to provide for a mathematically correct model for use in computer systems. Therefore, it seems more appropriate to look for a theoretical model of tolerancing, or tolerance representation scheme, that is in accordance with the international standards.

An early example of previous work in order to come to a mathematically correct model for tolerancing is the solids offset approach by Requicha [7], extended in [8]. In this approach, nominal surfaces are given a pair of offset surfaces to determine the tolerance zones. This approach differs from the tolerancing standards [9]. Because the individual pairs of offset surfaces are combined to obtain a composite tolerance zone of the entire solid, the individual tolerances cease to be independent constraints.

Jayaraman and Srinivasan introduced the notion of virtual boundary requirement in an attempt to redefine the notion of tolerances from the viewpoint of functional tolerances $[10,11]$. Jayaraman and Srinivasan note that the real purpose of tolerances is to characterize functional requirements. Two functional constraints are cited that are believed to account for most tolerances; the maintenance of material bulk in critical locations and spatial relationships for assembly. These requirements can be captured as virtual boundary requirements. Virtual Boundary Requirements are considered as a collection of virtual half spaces. The interpretation of datums in this approach is not in accordance with the standards.

Turner proposed a feasibility space approach $[12,13]$ which however does not seem to be suitable for 3D tolerance representation because of a too high complexity and doubtful usefulness in 3D.

Wirtz used a vectorial approach in which each tolerance is represented as a limit on the components of a vector that relates a given toleranced feature to a given reference feature [14]. The vectorial approach by Wirtz also seems insufficient: it is not close to the standards and is oriented too much towards older dimensioning and tolerancing practices.

Building on their earlier work in the field of computer aided inspection and the work by Requicha and Jayaraman and Srinivasan and Wirtz, Clément et al. arrive at a tolerance representation model which is compatible with the standards and which seems to be theoretically and mathematically sound [15-18]. Using the theory of the set of displacements by Hervé [19], Clément et al. have proven that there are only seven elementary surface types: spherical surface, planar surface, cylinder surface, helical surface, rotational surface, prismatic surface and "any" surface. When these seven surface types are combined, 28 cases of combination can be found. These combinations of surfaces are called TTRS: Technologically and Topologically Related Surfaces. There is a finite number $(\geq 44)$ of reclassifications of TTRS which denote the theoretical number of different tolerances (cases). On this basis, a computer system can automatically propose tolerance types employing an assernbly model (geometric tolerances). Also reference (datum) elements can be determined (semi-)automatically. These elements are called MGDE: Minimum Geometric Datum Element; see first two columns of Table 1 for the MGDEs related to each elementary surface as discerned by Clément et al. The same set of MGDE is used for determining the datum of composed TTRS. When two elementary surfaces are combined in a TTRS, the resulting TTRS can be classified into one of the seven basic classes depending on the types of surfaces involved and the geometric relations between them. See Table 1 for the reclassifications of TTRS. In this approach, tolerances are represented vectorially, in so-called torsors, allowing for tolerance analysis $[20,21]$. 
Table 1

Reclassifications of TTRS ${ }^{\text {a }}$

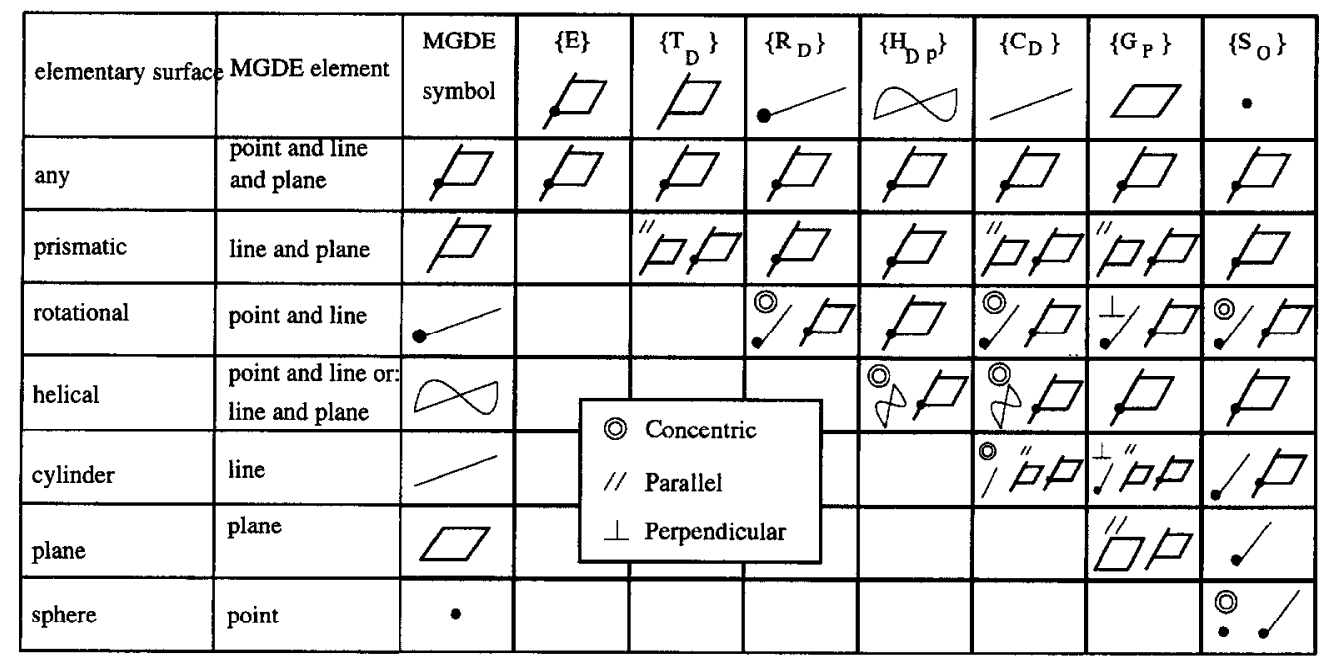

${ }^{a}$ The first two columns indicate the MGDE which is associated with each elementary surface. In the remaining table the cases of tolerancing between surfaces are indicated, including the resulting TTRS represented by their MGDEs (modified after [15])

\subsection{Previous work in tolerance specification}

A distinction is made between tolerance type specification, tolerance value specification and the use of manufacturing or process information in tolerance specification.

\subsubsection{Tolerance type specification}

The approach to tolerance specification as presented in [15-17], [22-25] is an exception to most previous approaches towards tolerance specification. Clément et al. propose a method to determine tolerance types automatically from the assembly model, resulting in a functional tolerance specification. Most other approaches in computer aided tolerancing are based on manual tolerance specification, usually starting out from single components. The tolerance specification method by Clément et al. is carried out on the basis of face associations between the different components in the assembly, also called the mating function [26]. By finding kinematic loops (in the graph representing the assembly), faces are found on individual components which can be toleranced relative to one another [25].

\subsubsection{Tolerance value specification}

Once tolerance types have been determined, either automatically or manually, tolerance values can be specified. So far, in existing literature tolerance value specification has not been automated. Of course a tolerancing system can propose some default tolerance values, but this does not reflect deep knowledge by the system on the functions to be performed. Clément et al. have made an investigation into different functions (like rotational guidance, translational guidance, etc.) and their relation to tolerance values using catalogues of large vendors of components like bearings [17]. From this research, however, it seems to be an overwhelming amount of work to develop a tolerance specification module which is able to automatically propose appropriate tolerance values in many different companies and application domains. However, a tolerance specification module which can be adapted according to the application domain and company and that is able to propose 


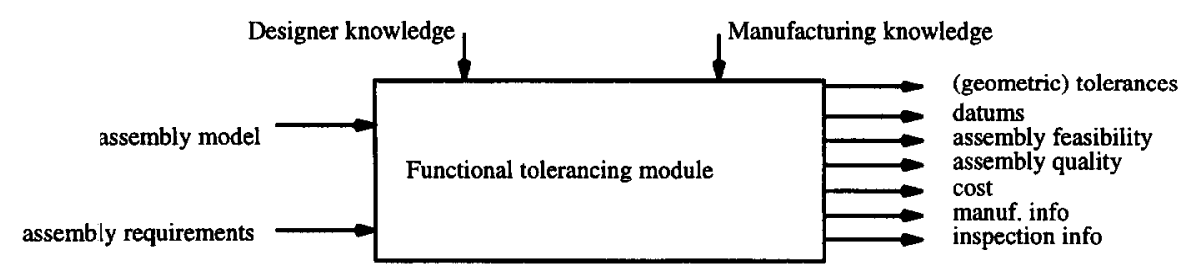

Fig. 2. Main function of the tolerancing module.

adequate tolerance values in most cases seems indeed feasible. Here, the system manager user as distinguished previously can fulfill a significant role. Nassef et al. employed genetic algorithms for tolerance value and type allocation [27]. However, this approach should be regarded as belonging to tolerance synthesis and less to tolerance specification.

\subsubsection{Use of manufacturing process / material information in tolerance specification}

If manufacturing process and/or material are known, this information can be used advantageously during tolerance specification. As such this can be seen as a kind of a priori tolerance synthesis. An example of this is provided in [28] in which process and material information are used in order to support the specification of size tolerances in the design of injection molded parts. A method is proposed that employs process simulation in order to assign optimal tolerances without constraining process engineers. Simulation is used to quantify the size tolerances due to process variations and estimate sensitivities. It is not clear whether the presented approach can easily be extended to include geometric tolerances together with size tolerances.

\section{Overall functionality of the tolerance specification tool}

A first design iteration of the design of the FROOM tolerance specification functionality has been described in [5,6]. Fig. 2 shows the main function of a tolerancing module in a simplified way. Fig. 2 also shows that apart from the tolerance types and values, a lot of additional data can be generated by a tolerancing module, e.g. on feasibility and quality of assembly, cost, etc. The areas of tolerance specification, analysis and synthesis can be

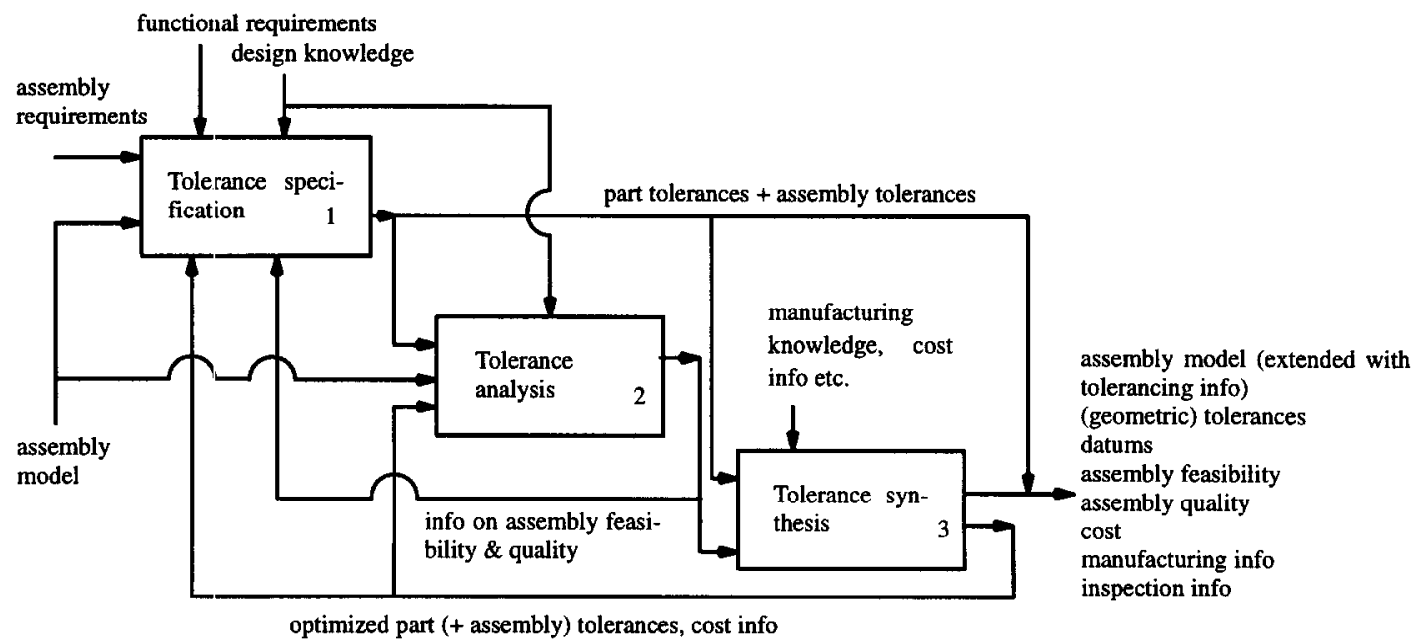

Fig. 3. The main sub-functions of the tolerancing module: tolerance specification, analysis and synthesis and their connections. 


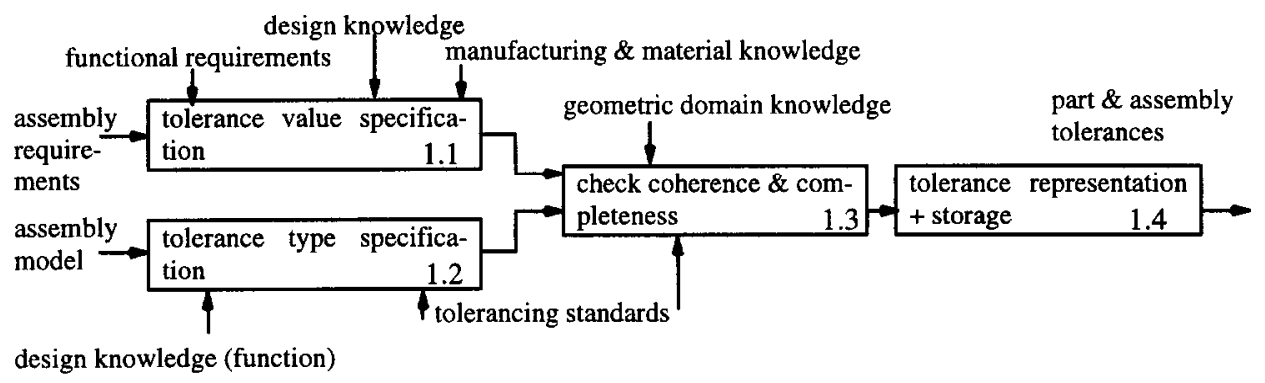

Fig. 4. Tolerance specification functionality (for simplicity retrieval and modification of existing tolerances as well as the iterative nature of tolerance specification is not shown).

seen as functions that need to be present in a re-design support system (Fig. 3). The functional decomposition of Fig. 3, was decomposed further resulting in the following sub functions: tolerance representation, tolerance specification, tolerance analysis, tolerance synthesis, tolerance presentation, storage and retrieval [5]. In the following only tolerance representation and tolerance specification are addressed in more detail.

1. For tolerance representation, i.e. the computer internal mathematical model of the tolerances, the vectorial torsor approach combined with the TTRS and MGDE concepts as described in e.g. $[16,17,29]$ has been selected. The main reason for this was that most other methods were not in conformance with the tolerancing standards. Another reason for selecting the torsor approach is that it is based on a strong mathematic background in kinematics, e.g. [19]. Using this mathematic background can offer great advantages in tolerance analysis and synthesis.

2. For tolerance specification, essentially the method as proposed by Clément et al. [15] was adopted, merely because there are no other methods for functional tolerance specification and because of the straightforward and logical nature of this approach. However, this method needs to be combined with a manual mode of tolerance specification as not all tolerances are by default functional in their origin.

The main sub-functions of the tolerance specification function of Fig. 3 are shown in Fig. 4.

\section{The selected tolerance representation scheme}

In the following the concepts of TTRS, MGDE and torsor are elaborated further as these are central to the tolerance representation in FROOM. Finally, the FROOM internal geometric tolerance representation format containing TTRS, MGDE and torsors is discussed.

\subsection{The applied TTRS concept}

In tolerancing, surfaces are often associated two by two. The seven elementary types of surfaces that were derived by Clément et al. can be associated two by two. The association of surfaces is central to the definition of TTRS (Technologically and Topologically Related Surfaces) as has been proposed by Clément et al. [15,26]:

A TTRS is defined as an assembly formed by two surfaces (or between a surface and a TTRS or between two TTRS) belonging to the same solid (topological aspect) and located in the same kinematic loop in a given mechanism (technological aspect).

A somewhat different, more generic, definition of TTRS is provided by Rivière [29]:

A TTRS is a pair of surfaces (or TTRS) belonging to the same solid which are associated because of functional reasons. 


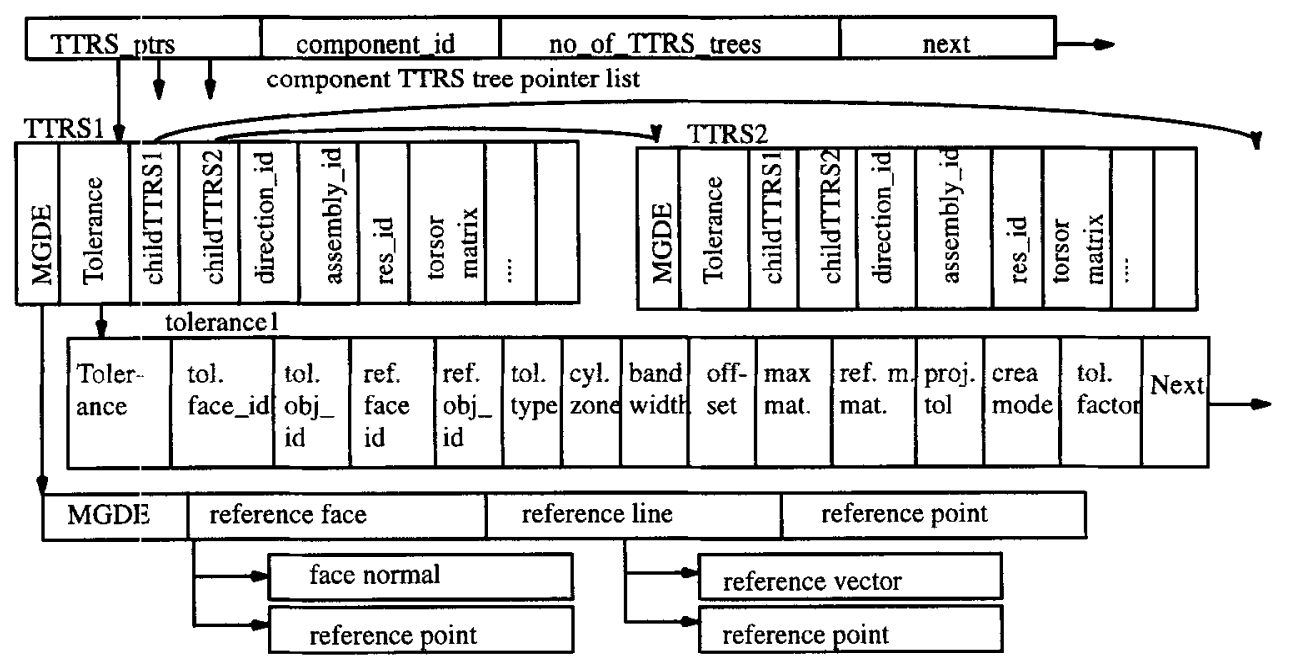

Fig. 5. Data structure for TTRS, MGDE and torsors in FROOM.

Clément et al. have made an extensive classification of all possible associations of the seven elementary surface types, and thus TTRS. One would expect $7 \times 7=49$ different types of association. However, only relative positions of two surfaces are of interest. Therefore, only 28 cases of surface association remain (i.e. $7+6+5+4+3+2+1$ cases or one half of a $7 \times 7$ matrix including the diagonal); see Table 1 . Although surface associations can be regarded as "symmetric", tolerances cannot. This is due to the fact that there is both a referenced and a toleranced face. Therefore, some tolerancing cases which are "below" the diagonal in Tablc 1 , and shown blank, should also be regarded [17].

When two elementary surfaces are combined in a TTRS, the resulting TTRS can be classified into one of the seven basic classes depending on the types of surfaces involved and the geometric relations between them. A systematic analysis of all possible cases of object reclassification has revealed (at least) 44 cases of tolerancing $[20,29]$. It must be noted here that the number of 44 was obtained by counting the number of cases in Table 1 and could grow if more special cases and cases below the diagonal are considered. Therefore, the actual number of the tolerancing cases is less important than the fact that there is only a finite number of tolerancing cases.

\subsection{The applied MGDE concept}

The Minimum Geometric Datum Element, or MGDE, of a TTRS is the minimum set of points, lines or planes necessary and sufficient to define the reference frame corresponding to the invariant sub-group of that TTRS. The concept of MGDE also has been proposed by Clément et al. [15,16]. The MGDE remains invariant for the displacement it is defining. The MGDE is a set of a reference point, reference line and a reference plane, but not all these elements are always necessary to define the MGDE sufficiently. The MGDE for a cylinder, for example, is the axis of the cylinder (i.e. the MGDE consists of a reference line only). Table 1 shows for each elementary surface its associated MGDE as well as the symbol used for the MGDE. In [15] rules are described that determine the location of MGDE.

\subsection{The applied torsor concept}

For each tolerance related to a TTRS, the tolerance zone can be represented as a tolerance torsor, which represents the small displacements that are possible within the tolerance zone. These small displacements are 
referred to as micro-degrees of freedom in order to make a distinction with large kinematic displacements, referred to as macro-degrees of freedom which can be used in solving geometric (nominal geometry) constraints [5,6] as well as in tolerance analysis; part II. Instead of torsors, matrices can be used for representing small displacements. The general displacement torsor/matrix can respectively be written as [21]:

$$
\begin{aligned}
T_{M, \theta} & =\left[\begin{array}{c}
\vec{D}_{M} \\
\vec{\theta}
\end{array}\right]=\left[\begin{array}{c}
u \\
v \\
w \\
\alpha \\
\beta \\
\gamma
\end{array}\right] \quad D(u, v, w, \alpha, \beta, \gamma) \\
& =\left[\begin{array}{cccc}
\gamma C \beta & -S \gamma C \alpha+C \gamma S \beta S \alpha & S \gamma S \alpha+C \gamma S \beta C \alpha & u \\
S \gamma C \beta & C \gamma C \alpha+S \gamma S \beta S \alpha & -C \gamma S \alpha+S \gamma S \beta C \alpha & v \\
-S \beta & C \beta S \alpha & C \beta C \alpha & w \\
0 & 0 & 0 & 1
\end{array}\right] .
\end{aligned}
$$

In this torsor/matrix $u, v$ and $w$ are the small translation parts along $x, y$ and $z$ axes respectively whereas $\alpha, \beta$ and $\gamma$ indicate the small rotations around these axes. For FROOM the matrix approach has been selected [6].

\subsection{Geometric tolerance representation implementation in FROOM}

The data structure in which both TTRS, MGDE and torsor matrix are stored in FROOM is shown in Fig. 5. This structure is stored in the Oracle ${ }^{T M}$ database. How TTRS, MGDEs and torsors are determined, is elaborated in [6].

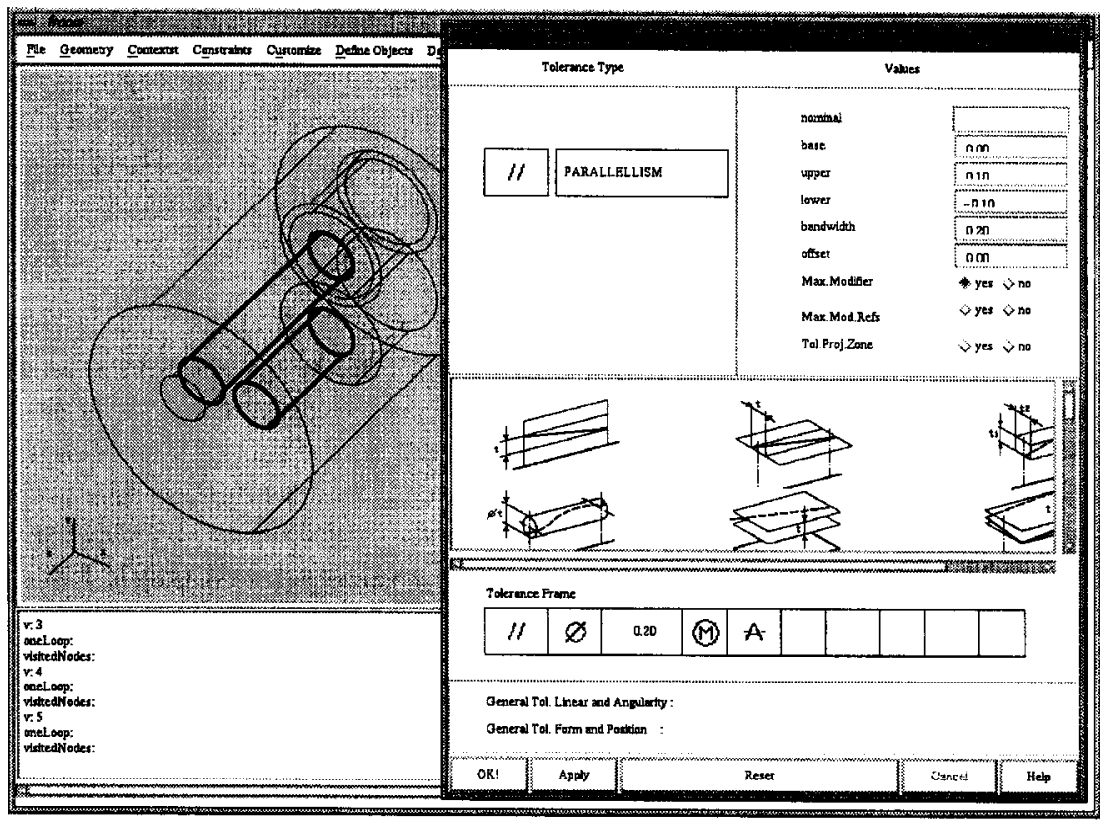

Fig. 6. The system proposes a parallelism tolerance between the two gear holes. 


\section{Semi-automatic tolerance specification in FROOM}

Based upon the theory as presented in [6] which on its turn is partly based upon [18,23,25], FROOM is able to generate tolerance types automatically based on a conceptual graph representation of an assembly model. The rules for loop detection, constructing MGDE, TTRS, etc, are implemented using $\mathrm{C}++$. Fig. 6 provides an example of tolerance specification of a gear pump. In this case the user has selected the option for automatic tolerance specification after assembly modeling was completed. Thus an assembly model with the nominal geometry of each component was necessary. From that moment the application is almost a one press button application. The user is proposed the tolerance types by the system based on the kincmatic loops and the TTRS theory. The user then only has to accept the tolerance type and give a tolerance value, offsets, etc.

\section{Manual tolerance specification}

Not all tolerances are by definition purely functional. Tolerances could for instance be specified in order to improve the visual appearance of an object. Also, users will always want to have the possibility to specify their "own"' tolerances or to overrule those proposed by the system.

In case of manual tolerance specification, it will be of great importance to also generate TTRS in order to allow downstream applications to work with the results from both semi-automatic and manual modes of tolerance specification. In fact, for the downstream applications the source of a specified tolerance (manual/automatic) should not matter. In an intermittent mode of manual and semi-automatic tolerance specification it is important to check for completeness and coherence of the specified tolerances.

The manual tolerance specification functionality has the user interface as shown in Fig. 7. On the lower left is the main tolerance specification window where the user decided what to do: manual tolerance specification or (semi-)automatic tolerance specification. Fig. 6 came into existence after clicking on the automatic specification option. In Fig. 7 on the lower right, the main window for manual tolerance specification is shown. A distinction has been made between the following options: manual specification of size tolerances, individual tolerances, related tolerances and assembly conditions (clearances or orientations). After this, the user is asked to select the face(s) to be toleranced. Once this has been done, the tolerance value, offset, etc. can be specified, most often in a window similar to the one of Fig. 6.

Size tolerances are subdivided into individual size tolerances (on a single face like a size tolerance on a diameter) and related size tolerances (always between 2 faces). Instead of specifying size tolerance values it is also possible to specify IT classes. Size tolerances are not transferred (yet) into geometric tolerances or TTRS. However, in tolerance analysis individual size tolerances are used to create some kind of a (pseudo) TTRS which however has faces on different components $[5,6]$. The term pseudo TTRS has been proposed by Clément et al. [30].

Individual tolerances are form tolerances, screw threads and roughnesses. Individual size tolerances are transformed into special TTRS (one single surface is transformed into a TTRS, the so-called TTRS cases 0-1 through $0-7$ [16]). Screw threads and roughnesses are specificd and stored for applications like process planning.

Related tolerances can be specified in two ways: either by just selecting the two faces to be toleranced or by selecting the two faces and the tolerance type. In the first case, the system uses the TTRS table (Table 1) to propose one or more tolerance types, similar to the semi-automatic specification. Thus, local incoherences can be avoided. However, the TTRS table does not provide for all types of tolerances that are available in the standards and that do have wide practical use. Examples are the runout and total runout tolerances. These tolerance types can be regarded as exceptions with respect to other tolerance types in that they are both orientation, position and form tolerances in one [23]. It is interesting to note that these tolerances mainly occur for cylindrical surfaces and surfaces of revolution of components which have at least one rotational (macro) 


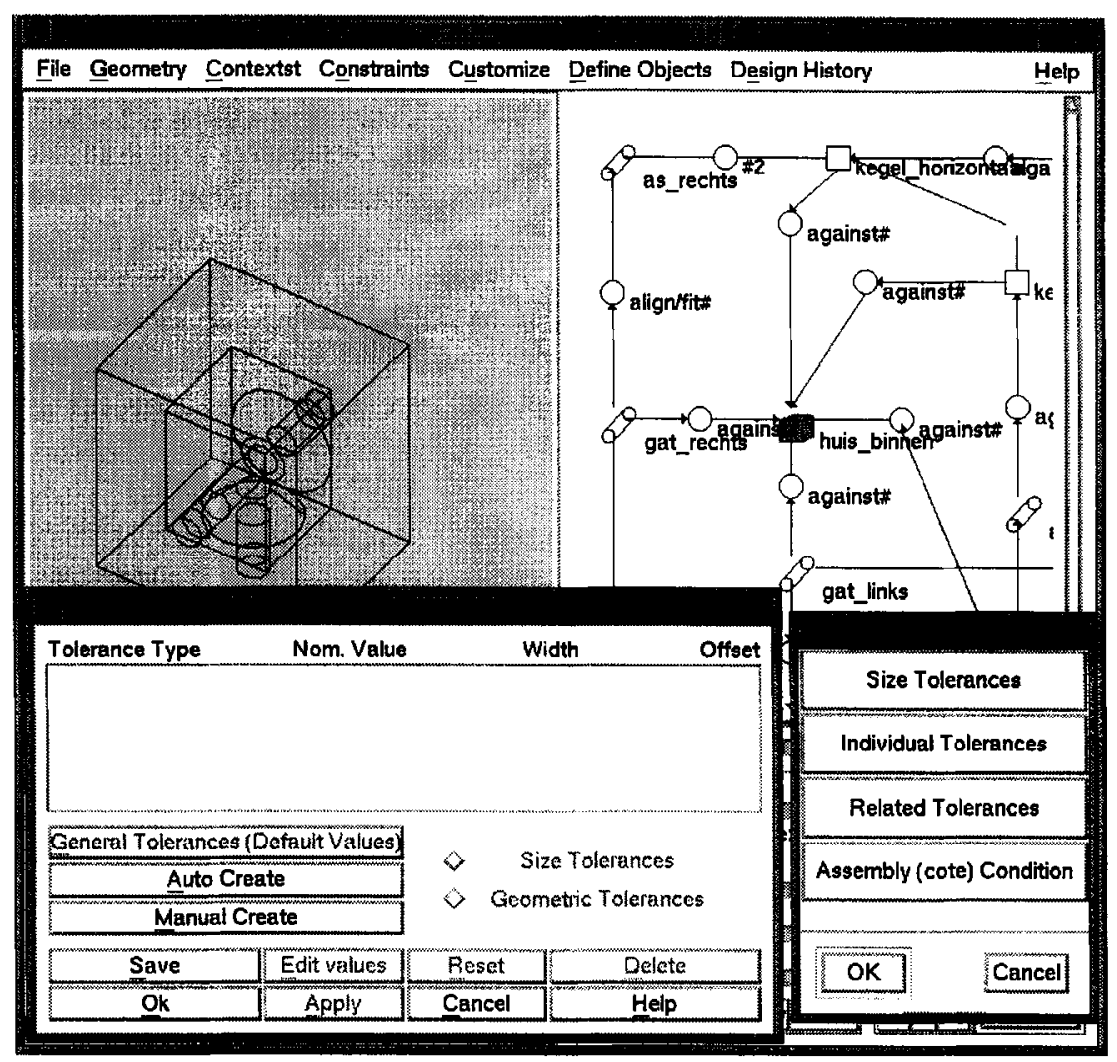

Fig. 7. Main tolerance specification window (lower left) with manual tolerance specification window which appears after clicking "manual create".

degree of freedom around their axes (representing a line basis-MGDE). This type of domain knowledge could easily be added to the knowledge contained in the TTRS table using a technique which keeps track of the macro-degrees of freedom of each component [5,6]. However, in order to overcome future omissions in the TTRS table, it is still possible in FROOM to specify a tolerance type and the two faces to be related by that tolerance type.

Assembly conditions are specified because clearances and orientations between faces of different components need also be specified.

Some of the data structures that are used in addition to those for geometric tolerances (which are represented by TTRS, MGDE and torsors) are shown in Fig. 8. These data structures are amongst others necessary for tolerance analysis, which is elaborated in part II on tolerance analysis.

Assembly (cote) condition

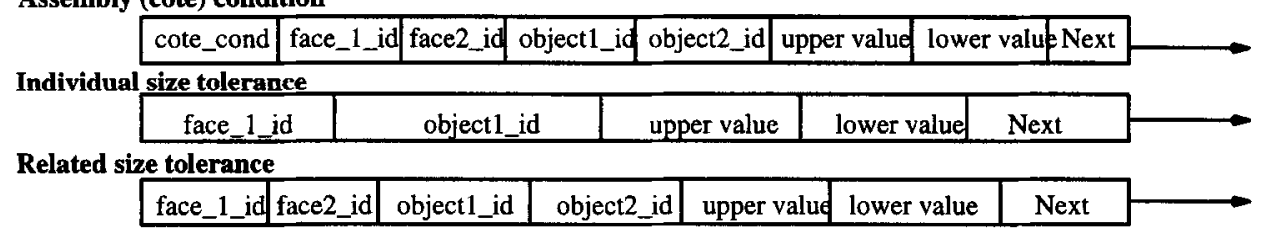

Fig. 8. Additional data structures for the manual tolerance specification functionality. 


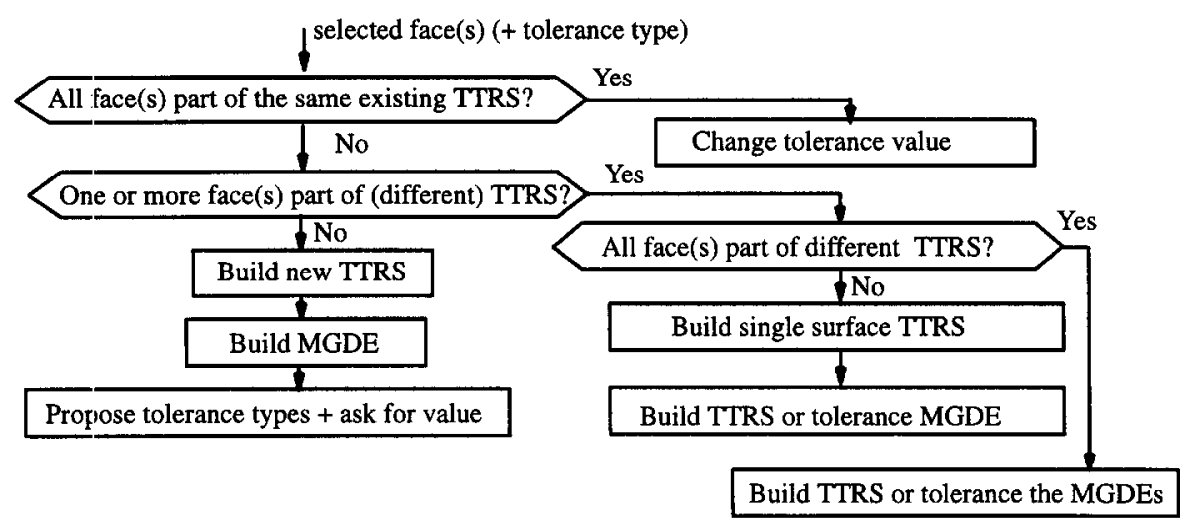

Fig. 9. Algorithm for manual tolerance specification.

\subsection{Building TTRS hierarchies in manual tolerance specification}

In the case of manual tolerance specification, there is not necessarily an assembly model whose kinematic loops together with some rules can help in building the TTRS hierarchies. Thus, some new rules seem necessary. In the following we propose some rules that help in manual tolerance specification.

In manual tolerance specification TTRS can be built if only form tolerances have been specified on single surfaces by using the 0-1 - 0-7 special TTRS cases. If related geometric tolerances have been specified between two surfaces normal TTRS can be built. In other cases data structures are built as indicated in Fig. 8 .

While building TTRS, first a check has to be performed whether all the selected face(s) are already part of one and the same existing TTRS/MGDE. If this is not the case, and if neither of the faces belongs to an existing TTRS, a completely new TTRS can be built using the TTRS table. In this case the system proposes tolerance types and the user has to determine the tolerance values. If, on the other hand, one or more of the selected faces are part of existing TTRS, the following cases can be distinguished. Either all selected faces are part of different previously existing TTRS: if one of the TTRS is of the "any" type, the MGDE of both TTRS should be toleranced. Otherwise TTRS can be built. This rule is in accordance with [18]. If one of the selected faces is part of a TTRS/MGDE and the other is not (it is "free"), then the surface not belonging to a TTRS should be transformed into a single surface TTRS. Then the same criterion as above should be applied. This approach is illustrated by Fig. 9 .

\subsection{Building TTRS hierarchies in mixed manual / automatic specification}

In a mixed mode of tolerance specification, both modes need to take into account TTRS results of the other mode. The semi-automatic mode for instance needs a check as described above and illustrated in Fig. 9 before new TTRS are built.

Once tolerance specification has been completed, in both modes, it is possible that for each component considered several unrelated TTRS trees exist. These TTRS can be related by tolerancing their MGDEs [18].

\section{Conclusions and recommendations}

A tolerance specification tool has been presented which allows for functional tolerance specification. The method focuses on the geometry necessary for fulfilling the required functions. Other aspects like lubrication, 
material or manufacturing process have not been considered. Semi-automatic tolerance specification, based on the method by Clément et al., has been combined with manual tolerance specification. In both cases a tolerance representation scheme is generated in terms of the TTRS model which is compatible with the tolerancing standards and which provides a correct mathematical model of tolerances, allowing for the computerization of downstream applications like tolerance analysis and synthesis.

Evaluation of the presented tolerance specification method in industrial practice is desirable. Extension to the support of the specification of tolerance values seems desirable. For the near future it seems most appropriate to follow a pragmatic approach in which the system manager gets some functionality to pre-define preferred default tolerance values depending on the particular application. The reason is that there is not yet a well understood general theory that describes the effect of tolerances on the behavior of mechanisms. As this understanding increases, tolerance specification functionality could be enhanced using information not only based on geometry, e.g. on lubrication, thermal effects, manufacturing process and material.

\section{Acknowledgements}

This research has been supported by the Technology Foundation (STW: a Dutch foundation).

\section{References}

[1] ISO 1101, Technical drawings - Geometrical tolerancing - Tolerances of form, orientation, location and run-out - Generalities, definitions, symbols, indications on drawings, 1983.

[2] ANSI Y 14.5M, Dimensioning and tolerancing, ASME, New York, 1994 (1982R).

[3] O.W. Salomons, F. van Slooten, G.W.F. de Koning, F.J.A.M. van Houten and H.J.J. Kals, “Conceptual graphs in CAD”, CIRP Annals, Vol. 43, No. 1, 1994, pp. 125-128.

[4] Spatial Technology, ACIS Geometric modeler, Boulder, Colorado, 1994.

[5] O.W. Salomons, "Computer support in the design of mechanical products, constraint specification and satisfaction in feature based design for manufacturing", Ph.D. Thesis, University of Twente, 1995.

[6] O.W. Salomons, H.J. Jonge Poerink, F. van Slooten, F.J.A.M. van Houten and H.J.J. Kals, "A tolerancing tool based on kinematic analogies", in: F. Kimura, ed., Computer Aided Tolerancing (Chapman and Hall, 1996).

[7] A.A.G. Requicha, "Toward a theory of geometric tolerancing”, International Journal of Robotics Research, Vol. 2, No. 4, 1983, pp. 45-59.

[8] A.A.G. Requicha and S.C. Chan, "Representation of geometric features, tolerances and attributes in solid modelers based on constructive geometry", IEEE Journal of Robotics and Automation, Vol. RA-2, No. 3, 1986, pp. 156-166.

[9] L.E. Farmer and C.A. Gladman, “Tolerance technology - Computer-based analysis”, Annals of the CIRP, Vol. 35, No. 1, 1986, pp. 7-10.

[10] R. Jayaraman and V. Srinivasan, "Geometric tolerancing I: Virtual boundary requirements", IBM Journal of Research and Development, Vol. 33, No. 2, 1989, pp. 90-104.

[11] V. Srinivasan and R. Jayaraman, “Geometric tolerancing: II. Conditional tolerances", IBM Journal of Research and Development, Vol. 33, No. 2, 1989, pp. 105-123.

[12] J.U. Turner and M.J. Wozny, "A mathematical theory of tolerances", in: M.J. Wozny and J.L. McLaughlin, eds., Geometric Modelling for CAD Applications, Elsevier Science Publishers, IFIP, 1988, pp. 163-187.

[13] J.U. Turner, "A feasibility space approach for automated tolerancing", ASME Journal of Engineering for Industry, Vol. 115, 1993, pp. 341-345.

[14] A. Wirtz, "Vectorial tolerancing a basic element for quality control", Proceedings of the 3rd CIRP Seminar on Computer Aided Tolerancing, Cachan (F), 1993, pp. 115-128.

[15] A. Clément, A. Desrochers and A. Rivière, "Theory and practice of 3D tolerancing for assembly", Proceedings of the CIRP Seminar on Computer Aided Tolerancing, Penn State University, U.S.A., May 1991.

[16] A. Clément and A. Rivière, "Tolerancing versus nominal modelling in next generation CAD/CAM system", Proceedings of the CIRP Seminar on Computer Aided Tolerancing, Paris, 1993, pp. 97-113.

[17] A. Clément, A. Rivière and M. Temmerman, Cotation tridimensionelle des systèmes mécaniques, théorie and pratique, PYC Edition, Yvry-Sur-Seine Cedex (ISBN 2-85330-132-X), 1994. 
[18] A. Desrochers and A. Clément, "A dimensioning and tolerancing assistance model for CAD/CAM systems", International Journal of Advanced Manufacturing Technology, Vol. 9, 1994, pp. 352-361.

[19] J.M. Hervé, "Analyse structurelle des mécanismes par groupe des déplacements", Mechanism and Machine Theory, Vol. 13, 1978, pp. $437-450$.

[20] D. Gaunet, "Modèle formel de tolerancement de position, contributions à l'aide au tolerancement des mécanismes en CFAO", Ph.D. Thesis, ENS de Cachan, 1994 (in French).

[21] A. Rivière, D. Gaunet, I. Dubé and A. Desrochers, “Une approche matricielle pour la représentation des zones de tolérance et des jeux", Proceedings, FORUM 1994 de la SCGM (Société Canadienne de Génie Mécanique), McGill University, 1994.

[22] B. Charles, A. Clérnent, A. Desrochers, P. Pelissou and A. Rivière, "Toward a computer aided tolerancing model", International Conference on CAD/CAM and AMT in Israël, CIRP Sessions on Tolerancing for Function in a CAD / CAM Environment, Vol. 2, Jerusalem, Israël, 1989.

[23] A. Desrochers, "Modèle conceptuel du dimensionnement et du tolérancement des mécanismes. Représentation dans les systèmes de CFAO", Ph.D. Thesis, École Centrale de Paris, 1991.

[24] A. Desrochers and R. Maranzana, "Constrained dimensioning and tolerancing assistance for mechanisms", Proceedings of the 4th CIRP Seminar on Computer Aided Tolerancing, Tokyo, 1995, pp. 21-29.

[25] P. Dufossé, "Automatic dimensioning and tolerancing", Prnceedings of the 3rd CIRP Seminar on Computer Aided Tolerancing, Cachan (F), 1993, pp. 1-10.

[26] M. Briard, B. Charles, A. Clément, P. Pelissou and A. Rivière, "The mating function in CAD/CAM systems", Design Automation Conference, Montreal, Canada, 1989.

[27] A. Nassef and H.A. ElMaraghy, "Allocation of tolerance types and values using genetic algorithms", Proceedings of the 3rd International CIRP Seminar on Computer Aided Tolerancing, ENS de Cachan, 1993, pp. 147-156.

[28] D.R. Busick, K.A. Beiter and K. Ishii, "Design for injection molding: Using process simulation to assess tolerance feasibility", Proceedings of the ASME Computers in Engineering Conference (CIE '94), Vol. 1, 1994, pp. 113-120.

[29] A. Rivière, "La géométrie du groupe des déplacements appliquée à la modélisation du tolerancement", Ph.D. Thesis, Paris, 1993.

[30] A. Clément, A. Rivière and P. Serré, "A declarative information model for functional requirements", Proceedings of the 4th CIRP Seminar on Computer Aided Tolerancing, Tokyo, 1995, pp. 1-20.

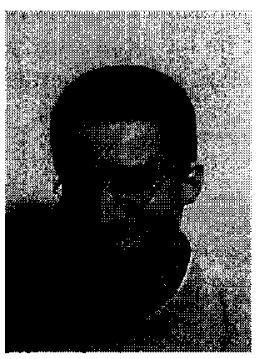

O.W. Salomons obtained a Ph.D. degree on his work on the FROOM system at the University of Twente in 1995.

He obtained his M.S. degree in Mechanical Engineering in 1990 at the same University. Presently, as an Assistant Professor, he is performing research in the field of design support systems as well as their link with process planning systems.

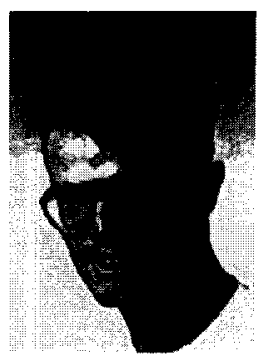

H.J. Jonge Poerink obtained his M.S. degree in Mechanical Engineering at the University of Twente in 1994. His M.S. work was on the tolerance specification module in the FROOM system. Currently he is employed at Philips Machinefabrieken. 


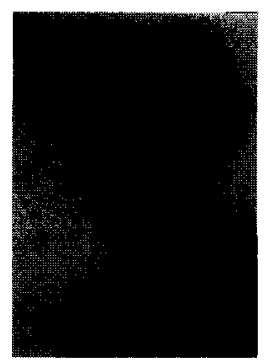

F.J. Haalboom obtained his M.S. degree in Mechanical Engineering at the University of Twente in 1995. His M.S. work was on the tolerance analysis module in the FROOM system. Currently he is employed at CAP-Volmac.
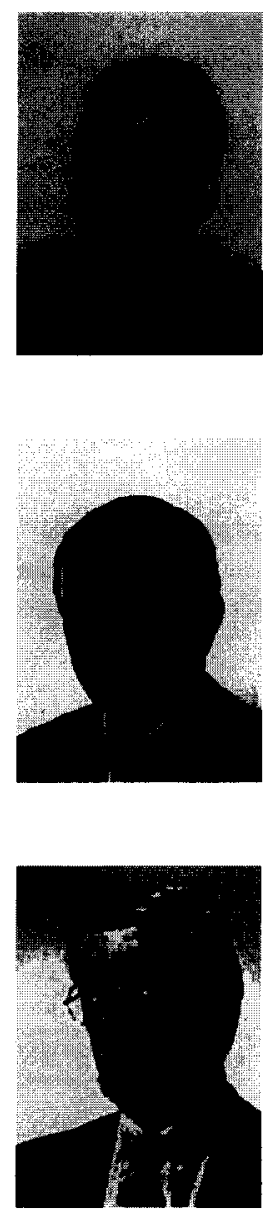

F. van Slooten holds a B.S. in Computer Science, which he obtained in 1990. Since 1991 he works as a System Analyst/ Technical Software Developer at the Laboratory of Production and Design Engineering of the University of Twente.
F.J.A.M. van Houten is Associate Professor of the Laboratory of Production and Design Engineering at the University of Twente. Professor Van Houten obtained his M.S. degree in Mechanical Engineering at the Technical University of Eindhoven in 1976. He has been working at the Laboratory of Production Enginecring at University of Twente since 1978. Professor Van Houten has worked in the field of CAD and CAPP; he has been closely involved with the development of several CAPP systems. In 1991 he obtained a Ph.D. degree on his work on the PART system.
H.J.J. Kals is Professor and Head of the Laboratory of Production Engineering at the University of Twente. He is also part-time Professor at the Technical University of Delft. Professor Kals obtained his M.S. degree in Mechanical Engineering in 1969 at the Technical University of Eindhoven. In 1972 he obtained his Ph.D. degree. In 1977 he became professor of the Laboratory of Production and Design Engineering at the University of Twente. Professor Kals is a member of CIRP. He is active in the fields of CAD, CAPP, CAM, workshop- and work station control. Currently one of his main scientific interests is concurrent engineering. 